\title{
On the Solution to the Plane Problem in the Static Theory of Elasticity for Multiply- Connected Regions with Given Surface Tractions
}

\section{A. D. FINE \& E. N. NILSON}

\section{Communicated by I. N. SNEDDoN}

1. Introduction. The complete analysis for the plane static problem in classical elasticity was first presented more than thirty years ago by N. I. Muskhelishvili [1]. Since then, many solutions have been obtained via this approach, but in almost all cases, these have involved either simply-connected, doubly-connected, or special multiply-connected regions. A method of solution for the general multiply-connected region was initially suggested by Sherman [2], but until now very little work has been done on this method. In this paper, we generalize Sherman's approach and show that the set of functional forms which he uses represents only a special case of the more general set of functional forms that we allow as being admissible.

2. Equations for multiply-connected regions. We consider a finite multiplyconnected plane region $S$, Figure 1 , and denote by $z=x+i y$ the complex representation of the point $(x, y)$ in the rectangular Cartesian coordinate system of the plane, with $i=\sqrt{-1}$. The displacements in the $x$-coordinate and in the $y$-coordinate directions are denoted by $u(x, y)$ and $v(x, y)$ respectively. The quantities $\sigma_{x}(x, y), \sigma_{y}(x, y)$, and $\tau_{x y}(x, y)$ are used to represent, respectively, the normal stress in the $x$ direction, the normal stress in the $y$ direction, and the tangential (shear) stress associated with these two directions. If we use the complex potential representation of Muskhelishvili [1], we obtain the following relations:

$$
\begin{gathered}
2 \mu\{u(x, y)+i v(x, y)\}=\frac{\lambda+3 \mu}{\lambda+\mu} \Phi(z)-z \overline{\Phi^{\prime}(z)}-\overline{\Psi(z)}, \\
\sigma_{x}(x, y)+\sigma_{y}(x, y)=2\left\{\Phi^{\prime}(z)+\overline{\Phi^{\prime}(z)}\right\}, \\
\sigma_{y}(x, y)-\sigma_{x}(x, y)-i 2 \tau_{x y}(x, y)=2\left\{\bar{z} \Phi^{\prime \prime}(z)+\Psi^{\prime}(z)\right\}, \\
155
\end{gathered}
$$


where $\lambda$ and $\mu$ are Lamé constants, $H^{\prime}(z)=d H(z) / d z$ for $H(z)$ any differentiable function of $z$, and $\bar{H}$ stands for the complex conjugate of $H$. The functions $\Phi(z)$ and $\Psi(z)$ are holomorphic (and consequently single-valved) in a simplyconnected region, but are only analytic (and therefore possibly multiply-valued) in a multiply-connected region. If we then differentiate equation (1) with respect to $z$ and look at the imaginary part of the resulting equation, we have:

$$
\frac{1}{2}\left\{\frac{\partial v(x, y)}{\partial x}-\frac{\partial u(x, y)}{\partial y}\right\}=\frac{\lambda+2 \mu}{\mu(\lambda+\mu)} \frac{1}{2 i}\left\{\Phi^{\prime}(z)-\overline{\Phi^{\prime}(z)}\right\}
$$

where the expression on the left-hand side of (4) represents the rotation at $z$ of an infinitesimal element of the body.

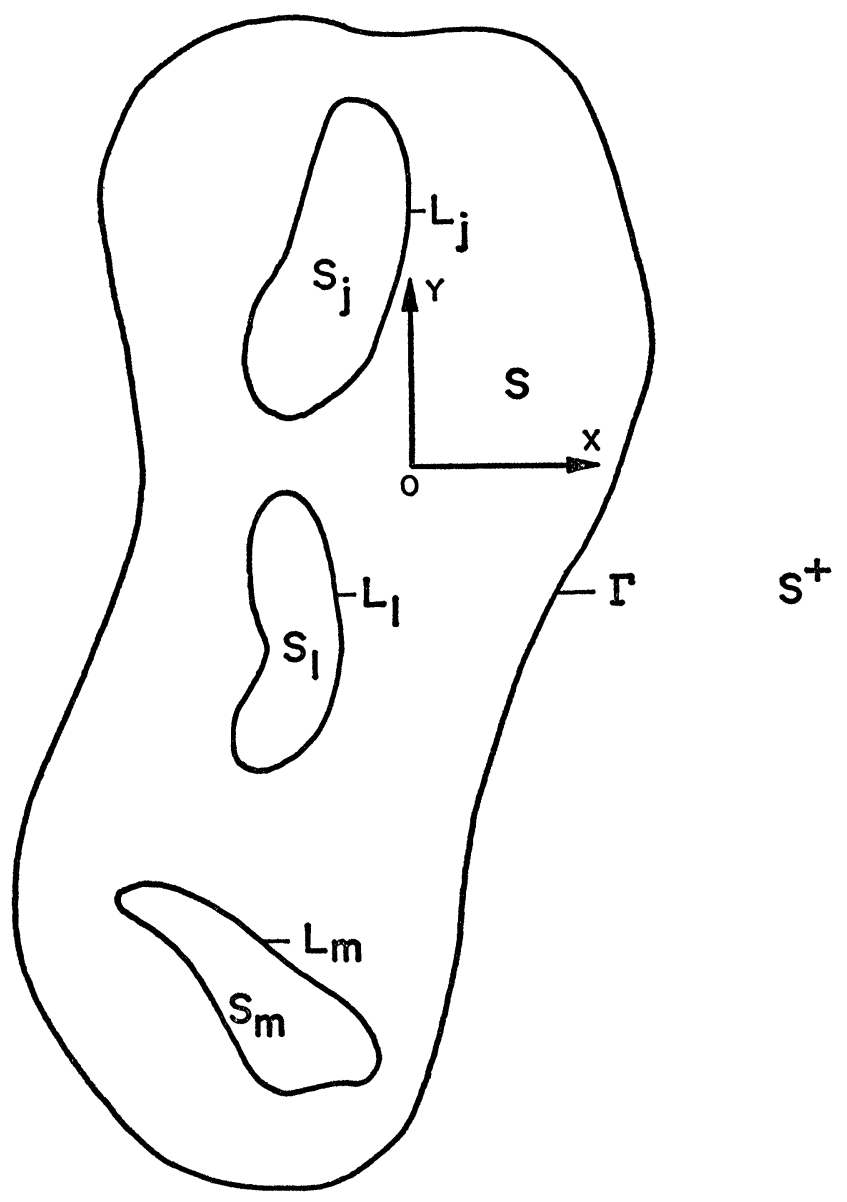

FigURE 1. General multiply-connected region. 
The region $S$ is bounded by a finite number of simple non-intersecting smooth contours $L_{1}, L_{2}, \cdots, L_{m}$, and $\Gamma$, the last of which contains all the others, Figure 1. We define by

$$
\partial S=L_{1}+L_{2}+\cdots+L_{m}+\Gamma
$$

the complete boundary of $S$. The finite region bounded by $L_{i}(j=1,2, \cdots m)$ is denoted by $S_{i}$, with $z_{j}$ an arbitrary point in $S_{i}$. The infinite region outside $\Gamma$ is designated as $S^{+}$, and for convenience we take the point $z=0$ to lie in $S$.

We are interested in the problem for which the surface tractions are specified over the entire boundary $\partial S$. The force equilibrium equations yield the following relation, which is valid both in $S$ and along $\partial S$ :

$$
i\left(X_{n}+i Y_{n}\right) d s=d\left\{\Phi(z)+z \overline{\Phi^{\prime}(z)}+\overline{\Psi(z)}\right\},
$$

where $X_{n}$ and $Y_{n}$ are the $x$ and $y$ components respectively of the surface traction acting on the differential arc length $d s$. In fact, equations (2) and (3) are derivable directly from (5).

By integrating equation (5) along the boundary $\partial S$, we obtain the relations

$$
\begin{aligned}
& \Phi(t)+\overline{t \Phi^{\prime}(t)}+\overline{\psi(t)}=F(t)+C_{i} \quad \text { on } \quad L_{i}, \quad j=1,2, \cdots, m, \\
& \Phi(t)+\overline{\Phi^{\prime}(t)}+\overline{\Psi(t)}=F(t) \quad \text { on } \quad \Gamma,
\end{aligned}
$$

where

$$
F(t)=i \int_{0}^{s}\left(X_{n}+i Y_{n}\right) d s
$$

on $L_{i}(j=1,2, \cdots m)$ and on $\Gamma$, with the arc coordinate $s$ measured positive in the counter-clockwise direction on each of the contours from an arbitrary fixed point of that contour, Figure 1 . The constants $C_{i}$, for $j=1,2, \cdots m$, have, in general, different values for different $L_{i}$ and are determined from the solution, but the constant of integration on the contour $\Gamma$ may be chosen arbitrarily and is taken to vanish.

Using the approach of Muskhelishvili, we consider the following functions for $z$ in $S$ and on $\partial S$ :

$$
\begin{aligned}
& \varphi(z)=\Phi(z)+\frac{1}{2 \pi(1+\nu)} \sum_{j=1}^{m}\left(X_{i}+i Y_{j}\right) \log \left(\frac{z-z_{i}}{z_{i}}\right), \\
& \psi(z)=\Psi(z)-\frac{\nu}{2 \pi(1+\nu)} \sum_{j=1}^{m}\left(X_{i}-i Y_{j}\right) \log \left(\frac{z-z_{j}}{z_{i}}\right),
\end{aligned}
$$

and for $t$ on $\partial S$ :

$$
\begin{aligned}
f(t)=F(t)+ & \frac{t}{2 \pi(1+\nu)} \sum_{i=1}^{m} \frac{\left(X_{i}-i Y_{j}\right)}{\left(t-\bar{z}_{j}\right)} \\
& +\frac{1}{2 \pi(1+\nu)} \sum_{i=1}^{m}\left(X_{i}+i Y_{i}\right)\left\{\log \left(\frac{t-z_{j}}{z_{i}}\right)-\nu \log \frac{\bar{t}-\bar{z}_{i}}{\bar{z}_{i}}\right\},
\end{aligned}
$$


where $\nu=(\lambda+3 \mu) /(\lambda+\mu)$ and the quantities $X_{i}$ and $Y_{i}$ are the resultant components in the $x$ and $y$ directions respectively of the prescribed surface tractions applied to the contour $L_{i}$.

If we then substitute relations (8) and (9) into (6), we obtain

$$
\begin{aligned}
& \varphi(t)+\overline{t \varphi^{\prime}(t)}+\overline{\psi(t)}=f(t)+C_{i} \quad \text { on } L_{i}, \quad j=1,2, \cdots, m, \\
& \varphi(t)+\overline{t \varphi^{\prime}(t)}+\overline{\psi(t)}=f(t) \quad \text { on } \Gamma .
\end{aligned}
$$

The final equilibrium condition that must be imposed expresses the balance of moments produced by the surface tractions acting on $\partial S$. This reduces, with the aid of (9), to the following equation:

$$
\oint_{\partial S}\{f(t) \overline{d t}+\overline{f(t)} d t\}=0
$$

where the symbol $\mathscr{\swarrow}_{\partial S}$ is defined by

$$
\oint_{\partial S}=\oint_{\Gamma}-\sum_{j=1}^{m} \oint_{L_{i}}
$$

The notation $\mathscr{S}_{L_{f}}\left(\mathscr{S}_{\mathrm{r}}\right)$ indicates an integration in the counter-clockwise direction once around the contour $L_{i}(\Gamma)$.

The analysis presented by Muskhelishvili [1] shows that the functions $\varphi(z)$ and $\psi(z)$ as defined in (8) are each holomorphic in $S$. From equations (7) and (9), we deduce that $f(t)$ is single-valued and continuous on each contour $L_{i}$ as well as on $\Gamma$. If we now recall the Cauchy integral representation for holomorphic functions, we see that the problem reduces to finding $\varphi(t)$ and $\psi(t)$ on $\partial S$.

3. Generalization of the method of Sherman. A method for solving $\varphi(t)$ and $\psi(t)$ in (10), subject to condition (11), was first proposed by Sherman [2] and is presented essentially unchanged by Muskhelishvili [1]. Our generalization of this approach starts with a consideration of the following integral equation on the boundary $\partial S$ :

$$
\begin{aligned}
& \text { (12) } \begin{aligned}
& \omega(t)+\frac{1}{2 \pi i} \oint_{\partial S} \omega(\tau) d \log \frac{\tau-t}{\bar{\tau}-\bar{t}}-\frac{1}{2 \pi i} \oint_{\partial S} \overline{\omega(\tau)} d \frac{\tau-t}{\bar{\tau}-\bar{t}} \\
&+\sum_{i=1}^{m}\left\{\frac{b_{i}}{t-\zeta_{i}}+\frac{\bar{b}_{j}}{\bar{t}-\bar{\zeta}_{i}}\left(1-\frac{t}{\bar{t}-\bar{\zeta}_{i}}\right)\right\}+ \frac{b_{m+1}}{t-a}+\frac{\bar{b}_{m+1}}{t-\bar{a}}\left\{1-\frac{t-a}{\bar{t}-\bar{a}}\right\}-C_{i} \\
&=f(t) \quad \text { on } \quad L_{i}, \quad j=1,2, \cdots, m
\end{aligned}
\end{aligned}
$$

and 
(13)

$$
\begin{aligned}
& \omega(t)+\frac{1}{2 \pi i} \oint_{\partial S} \omega(\tau) d \log \frac{\tau-t}{\bar{\tau}-\bar{t}}-\frac{1}{2 \pi i} \oint \overline{\omega(\tau)} d \frac{\tau-t}{\bar{\tau}-\bar{t}} \\
& +\sum_{i=1}^{m}\left\{\frac{b_{j}}{t-\zeta_{i}}+\frac{\bar{b}_{j}}{\overline{t-\bar{\zeta}_{i}}}\left(1-\frac{t}{\bar{t}-\bar{\zeta}_{i}}\right)\right\}+\frac{b_{m+1}}{t-a}+\frac{\bar{b}_{m+1}}{\bar{t}-\bar{a}}\left\{1-\frac{t-a}{\bar{t}-\bar{a}}\right\} \\
& =f(t) \text { on } \Gamma,
\end{aligned}
$$

with equations (12) and (13) subject to condition (11).

The function $\omega(t)$ is assumed to have a continuous derivative $\omega^{\prime}(t)$ on $\partial S$. The constants $b_{i}$ are taken to be real functionals of $\omega(t)$ on the corresponding contours $L_{i}$ so that we say

$$
b_{i}[\omega(t)]=B_{i}[\omega(t)]+\overline{B_{i}[\omega(t)]}, \quad j=1,2, \cdots, m,
$$

where $B_{i}[\omega(t)]$ is a linear functional of $\omega(t)$ on $L_{j}$. The constant $b_{m+1}$ is assumed to be a pure imaginary functional of $\omega(t)$ on $\partial S$ and we have

$$
b_{m+1}[\omega(t)]=B[\omega(t)]-\overline{B[\omega(t)]},
$$

where $B[\omega(t)]$ is a linear functional of $\omega(t)$ on $\partial S$. The constants $C_{i}$ are hypothesized as being linear functionals of $\omega(t)$ on the corresponding $L_{j}$. The locations $z=\zeta_{i}$, for $j=1,2, \cdots m$, are arbitrary points in the corresponding regions $S_{i}$, while the location $z=a$ is an arbitrary point in $S$.

We then define the functions $\varphi(z)$ and $\psi(z)$ for $z$ in $S$ as follows:

$$
\begin{aligned}
& \varphi(z)=\frac{1}{2 \pi i} \oint_{\partial S} \frac{\omega(\tau) d \tau}{\tau-z}+\sum_{i=1}^{m} \frac{b_{i}}{z-\zeta_{i}}, \\
& \psi(z)=\frac{1}{2 \pi i} \oint_{\partial S} \frac{\overline{\omega(\tau)}-\bar{\tau} \omega^{\prime}(\tau)}{\tau-z} d \tau+\sum_{i=1}^{m} \frac{b_{i}}{z-\zeta_{i}} .
\end{aligned}
$$

It is clear that each of these two functions is holomorphic for $z$ in $S$. With the aid of the Plemelj formulae [1] we find, from equations (16) and (17), the boundary values $\varphi(t), \varphi^{\prime}(t)$, and $\psi(t)$ on $\partial S$. Substituting these expressions in equations (12) and (13), we obtain

$$
\begin{gathered}
\varphi(t)+\overline{t \varphi^{\prime}(t)}+\overline{\psi(t)}+b_{m+1}\left\{\frac{1}{t-a}-\frac{1}{t-\bar{a}}+\frac{t-a}{(\bar{t}-\bar{a})^{2}}\right\}-C_{i} \\
=f(t) \quad \text { on } L_{i}, \quad j=1,2, \cdots, m \\
\varphi(t)+\overline{t \varphi^{\prime}(t)}+\overline{\psi(t)}+b_{m+1}\left\{\frac{1}{t-a}-\frac{1}{\bar{t}-\bar{a}}+\frac{t-a}{(\bar{t}-\bar{a})^{2}}\right\}=f(t) \text { on } \Gamma .
\end{gathered}
$$

Applying condition (11) to equations (18) and (19), using integration by parts, and recalling that $b_{m+1}$ is pure imaginary, we find that

$$
b_{m+1}[\omega(t)]=0 .
$$


We conclude therefore, as did Sherman, that any solution to equations (18) and (19), and consequently to equations (12) and (13), subject to condition (11), must also be a solution for equation (10), subject to condition (11). In fact, the solutions for $\varphi(z)$ and $\psi(z)$ can differ only by terms which give a rigid body displacement.

Equations (12) and (13) can be separated into a system of two Fredholm equations for two unknowns, the real and imaginary parts of $\omega(t)$ on $\partial S$. Since the Fredholm Alternative is applicable here, we can prove both the existence as well as the uniqueness of solutions for equations (12) and (13), subject to condition (11), by showing that the homogeneous equations obtained when $f(t)=0$ (zero surface traction case) have only the null solution. In this situation, all the stresses vanish and, as was shown by Muskhelishvili [1], we find that $\varphi(z), \psi(z)$, and $C_{i}$ are given as follows:

$$
\begin{gathered}
\varphi_{0}(z)=i_{\epsilon z}+c, \\
\psi_{0}(z)=-\bar{c}, \\
C_{i}\left[\omega_{0}(t)\right]=0, \quad j=1,2, \cdots, m,
\end{gathered}
$$

for $z$ in $S$. The zero subscript indicates here and in subsequent equations the solution to the zero stress problem in $S$.

Substituting these expressions, (21) and (22), into equations (16) and (17), we arrive at the following relations valid for $t$ on $\partial S$ :

$$
\begin{aligned}
& i \varphi^{*}(t)=\omega_{0}(t)-i \epsilon t-c+\sum_{i=1}^{m} \frac{b_{j}\left[\omega_{0}(t)\right]}{t-\zeta_{i}}, \\
& i \psi^{*}(t)=\overline{\omega_{0}(t)}-\bar{t} \omega_{0}^{\prime}(t)+\bar{c}+\sum_{i=1}^{m} \frac{b_{i}\left[\omega_{0}(t)\right]}{t-\zeta_{j}},
\end{aligned}
$$

where $\varphi^{*}(t)$ and $\psi^{*}(t)$ are the boundary values of the functions $\varphi^{*}(z)$ and $\psi^{*}(z)$ respectively which are holomorphic and single valued in the regions $S_{1}, S_{2}, \cdots S_{m}$, and $S^{+}$. In addition, both $\varphi^{*}(z)$ and $\psi^{*}(z)$ approach zero as $z$ approaches infinity in $S^{+}$.

Multiplying equation (25) by $d t$, integrating separately over each of the contours $L_{i}$, and using integration by parts, we arrive at the result that

$$
b_{j}\left[\omega_{0}(t)\right]=0, \quad j=1,2, \cdots, m,
$$

where the assumption that the $b_{j}$ are real has been used. We insert (26) into expressions (24) and (25) and eliminate $\omega_{0}(t)$ from the resulting equation This yields the following equation valid for $t$ on $\partial S$ :

$$
\varphi^{*}(t)+\overline{t \varphi^{*^{\prime}}(t)}+\overline{\psi^{*}(t)}=2 i c-2 \epsilon t .
$$

We then consider equation (27) separately on each of the contours $L_{i}$. It is seen that $\varphi^{*}(t)$ and $\psi^{*}(t)$ solve the plane elastic boundary value problem in $S_{i}$ for the case when a uniform hydrostatic pressure of magnitude $\epsilon$ is applied 
to the boundary $L_{i}$ of $S_{i}$. We can therefore find both $\varphi^{*}(t)$ and $\psi^{*}(t)$ on each of the contours $L_{i}$ for $j=1,2, \cdots m$, as follows:

$$
\begin{aligned}
& \varphi^{*}(t)=\left(i \epsilon_{i}-\epsilon\right) t+2 i c+h_{j}, \\
& \psi^{*}(t)=-\bar{h}_{j},
\end{aligned}
$$

where $\epsilon_{i}$ are real constants and $h_{j}$ are complex constants. Combining (28) with (24) and using (26), we have that

$$
\omega_{0}(t)=-\epsilon_{j} t+\left(i h_{i}-c\right) \quad \text { on } L_{i}, \quad j=1,2, \cdots, m .
$$

We now put further restrictions on the functional forms $B_{j}[\omega(t)]$ and $C_{j}[\omega(t)]$ :

$$
\begin{aligned}
& B_{i}[\omega(t)]+\overline{B_{i}[\omega(t)]} \neq 0 \quad \text { if } \omega(t)=t \quad \text { on } L_{i}, \\
& B_{i}[\omega(t)]=0 \quad \text { if } \quad \omega(t)=1 \quad \text { on } L_{i} \text {, } \\
& C_{i}[\omega(t)] \neq 0 \quad \text { if } \omega(t)=1 \text { on } L_{i} \text {. }
\end{aligned}
$$

By then applying restrictions (26) and (23) to equation (30) and using these assumptions, (31)-(33), we can make $\omega_{0}(t)$ vanish on each of the inner contours $L_{i}$ so that

$$
\omega_{0}(t)=0 \quad \text { on } \quad L_{i}, \quad j=1,2, \cdots, m .
$$

Recalling condition (20) and expression (15), we obtain

$$
B[\omega(t)]-\overline{B[\omega(t)]}=0 .
$$

We now split up $B[\omega(t)]$ as follows:

$$
B[\omega(t)]=B_{\Gamma}[\omega(t)]+\sum_{j=1}^{m} R_{j}[\omega(t)],
$$

where $B_{\Gamma}$ is a linear functional of $\omega(t)$ on $\Gamma$ and $R_{i}$ is a linear functional of $\omega(t)$ on $L_{i}, j=1,2, \cdots m$. For the zero stress case, where we have just shown that $\omega_{0}(t)$ vanishes on each of the inner contours, equation (35) becomes, with the aid of (34) and (36),

$$
B_{\Gamma}\left[\omega_{0}(t)\right]-\overline{B_{\Gamma}\left[\omega_{0}(t)\right]}=0 .
$$

We then apply condition (37) to expression (24). By restricting the functional form of $B_{\mathrm{r}}$ such that

$$
B_{\Gamma}[\omega(t)]=0,
$$

if $\omega(t)$ is the boundary value on $\Gamma$ of a function analytic in $S^{+}$and at infinity,

$$
B_{\Gamma}[\omega(t)]+\overline{B_{\Gamma}[\omega(t)]} \neq 0,
$$

if $\omega(t)=t$ on $\Gamma$, we obtain the result that

$$
\epsilon=0 .
$$


Using (40) in (27) and recalling that $\varphi^{*}(t)$ and $\psi^{*}(t)$ are the boundary values on $\Gamma$ of functions analytic in $S^{+}$and at infinity, and approaching zero as $z$ approaches infinity, we see that on $\Gamma$ equation (27) with $\epsilon=0$ represents a plane elastic problem for zero surface tractions applied to the boundary $\Gamma$ of the infinite region $S^{+}$. We conclude therefore that both $\varphi^{*}(z)$ and $\psi^{*}(z)$ must vanish for $z$ in $S^{+}$, that $c=0$, and finally that $\omega_{0}(t)=0$ on $\Gamma$. Thus $\omega_{0}(t)$ is zero for every $t$ on $\partial S$ and our proof is complete.

4. Arbitrariness of the functional forms. The question now arises as to what effect, if any, the choice of functional forms, subject to the restrictions given in the previous section, can have on the final solution to $\omega(t)$, or more importantly, what effect such choices can have on the solutions to $\varphi(z)$ and $\psi(z)$. We begin with the premise that the functional dependence of $b_{m+1}$ on $\omega(t)$ is unaffected by any change in the functional dependence of $b_{i}$ or $C_{i}$ on $\omega(t)$.

We assume that ${ }^{\prime} b_{m+1}[\omega(t)]$ and ${ }^{\prime \prime} b_{m+1}[\omega(t)]$ are two different possible functionals for $b_{m+1}$, and that ' $\omega(t),{ }^{\prime} \varphi(z),{ }^{\prime} \psi(z)$, and ${ }^{\prime \prime} \omega(t),{ }^{\prime \prime} \varphi(z),{ }^{\prime} \psi(z)$ are the corresponding solutions. By carrying out an analysis similar to that given in Section 3, we readily see that these two sets of solutions can differ only in the following way:

$$
\begin{aligned}
\prime \prime \omega(t)-{ }^{\prime} \omega(t) & =0 \quad \text { on } L_{i}, \quad j=1,2, \cdots, m, \\
\prime \prime \omega(t)-{ }^{\prime} \omega(t) & =Q^{*}(t)+i \alpha t+\beta \text { on } \Gamma, \\
{ }^{\prime \prime} \varphi(z)-{ }^{\prime} \varphi(z) & =i \alpha z+\beta, \\
\prime \prime \psi(z)-' \psi(z) & =-\bar{\beta},
\end{aligned}
$$

where $Q^{*}(t)$ is the boundary value on $\Gamma$ of a function $Q^{*}(z)$ homomorphic in $S^{+}$and approaching zero as $z$ approaches infinity, and where $\alpha$ is a real and $\beta$ a complex constant, with $\alpha$ given by

$$
\alpha=-\frac{i^{\prime} b_{m+1}\left[{ }^{\prime \prime} \omega(t)\right]}{{ }^{\prime} B_{\Gamma}[t]+{ }^{\prime} B_{\Gamma}[t]}=\frac{i^{\prime \prime} b_{m+1}\left[{ }^{\prime} \omega(t)\right]}{{ }^{\prime \prime} B_{\Gamma}[t]+{ }^{\prime \prime} \bar{B}_{\Gamma}[t]} .
$$

We therefore conclude that the choice of a functional form for $b_{m+1}[\omega(t)]$ does not affect the solution for $\omega(t)$ on the inner contours $L_{i}$, but does change the solution for $\omega(t)$ quite drastically on $\Gamma$ by a function which is the boundary value of a function analytic in $S^{+}$and at infinity as well as by a function linear in $t$ (42). The final solutions for $\varphi(z)$ and $\psi(z)$, however, are modified by terms which give only a rigid body displacement.

We next consider what effect the functional form of $C_{j}[\omega(t)]$ has on the solutions for $\omega(t), \varphi(z)$, and $\psi(z)$. To facilitate the analysis, let us define the operator $P[\omega(t)]$ for $t$ on $\partial S$ by 
(46)

$$
\begin{aligned}
& P[\omega(t)]=\omega(t)+\frac{1}{2 \pi i} \oint_{\partial S} \omega(\tau) d \log \frac{\tau-t}{\bar{\tau}-t} \\
& -\frac{1}{2 \pi i} \oint_{\partial S} \overline{\omega(\tau)} d \frac{\tau-t}{\bar{\tau}-t}+\sum_{i=1}^{m} b_{j}[\omega(t)]\left\{\frac{1}{t-\zeta_{i}}+\frac{1}{t-\bar{\zeta}_{i}}-\frac{t}{\left(t-\bar{\zeta}_{i}\right)^{2}}\right\} .
\end{aligned}
$$

Thus, equations (12), (13), and (20) can be written as

$$
\begin{aligned}
P[\omega(t)]-C_{j}[\omega(t)] & =f(t) \quad \text { on } \quad L_{i}, \quad j=1,2, \cdots, m, \\
P[\omega(t)] & =f(t) \quad \text { on } \Gamma, \\
b_{m+1}[\omega(t)] & =0 \quad \text { on } \partial S .
\end{aligned}
$$

We look at any two possible sets of functionals for $C_{j}[\omega(t)]$, say $M_{j}[\omega(t)]$ and $N_{j}[\omega(t)]$, and let $\omega^{M}(t), \varphi^{M}(z), \psi^{M}(z)$ and $\omega^{N}(t), \varphi^{N}(z)$, and $\psi^{N}(z)$ be the corresponding solutions. The difference between the solutions for $\omega(t)$,

$$
D(t)=\omega^{N}(t)-\omega^{M}(t),
$$

is easily seen to obey the following relations:

$$
\begin{aligned}
P[D(t)]-N_{j}[D(t)] & =-A_{i} \quad \text { on } \quad L_{i}, \quad j=1,2, \cdots, m, \\
P[D(t)] & =0 \quad \text { on } \Gamma, \\
b_{m+1}[D(t)] & =0 \quad \text { on } \partial S,
\end{aligned}
$$

where the $A_{i}$ are defined by

$$
A_{i}=\left(M_{i}-N_{i}\right)\left[\omega^{M}(t)\right] \quad \text { on } \quad L_{i}, \quad j=1,2, \cdots, m .
$$

We can now consider equations (50)-(52) as a set of integral equations for $D(t)$. This set, of course, has a unique solution; and by using the method employed in the previous section, we can show that $D(t)$ takes the form,

$$
\begin{aligned}
& D(t)=d_{j}=\frac{A_{i}}{N_{i}[1]} \text { on } L_{i}, \quad j=1,2, \cdots, m, \\
& D(t)=0 \text { on } \Gamma,
\end{aligned}
$$

when

$$
\sum_{j=1}^{m}\left\{d_{i} R_{j}[1]-\overline{d_{i} R_{i}[1]}\right\}=0 .
$$

Thus, in this case, a change in the functional form of $C_{j}[\omega(t)]$ will produce a change in $\omega(t)$ by only a constant on each of the inner contours $L_{i}$. Consequently, for such a situation, $\varphi(z)$ and $\psi(z)$ would both remain unchanged. It is interesting to note, in passing, that if further restrictions were placed on $b_{m+1}[\omega(t)]$ such that

$$
R_{i}[\omega(t)]=0 \text { if } \omega(t)=1 \text { on } L_{i},
$$

then equation (55) would also be satisfied. 
Finally, we assert without proof, although the proof is fairly straightforward, that the choice of functional forms for $b_{j}[\omega(t)]$, subject to restrictions (31) and (32), can have no effect on the final solution for $\omega(t)$ and consequently no effect on the solution for either $\varphi(z)$ or $\psi(z)$. In addition, the choice of points $\rho_{i}$ in $S_{i}$ and of the point $a$ in $S$ also has no effect on the final results.

5. Discussion and comparison of results. The particular functional forms that Sherman [2] uses are as follows:

$$
\begin{aligned}
B_{\Gamma}[\omega(t)] & =\frac{1}{2 \pi i} \oint_{\Gamma} \frac{\omega(t) d t}{t^{2}}, \\
R_{i}[\omega(t)] & =-\frac{1}{2 \pi i} \oint_{L_{i}} \frac{\omega(t) d t}{t^{2}}, \\
B[\omega(t)] & =\frac{1}{2 \pi i} \oint_{\partial S} \frac{\omega(t) d t}{t^{2}}, \\
b_{m+1}[\omega(t)] & =\frac{1}{2 \pi i} \oint_{\partial S}\left\{\frac{\omega(t) d t}{t^{2}}+\frac{\overline{\omega(t)}}{\bar{d}} \overline{\bar{t}^{2}}\right\}, \\
B_{i}[\omega(t)] & =i \oint_{L_{i}} \omega(t) \overline{d t} \\
b_{j}[\omega(t)] & =i \oint_{L_{i}}\{\omega(t) \overline{d t}-\overline{\omega(t)} d t\}, \\
C_{i}[\omega(t)] & =-\oint_{L_{i}} \omega(t) d s .
\end{aligned}
$$

It is important to note that expression (58) obeys condition (56), although, as we have shown, this restriction (56) is not needed for the existence and uniqueness proof. We conclude therefore that, in Sherman's method, any change in the functional form for $C_{i}[\omega(t)]$ will leave the results for $\varphi(z)$ and $\psi(z)$ completely unaltered.

If we reexamine our initial equilibrium equations, (10) and (11), we know that the solution for the zero surface traction case is

$$
\begin{aligned}
& \varphi_{0}(z)=i_{\epsilon} z+c, \\
& \psi_{0}(z)=-\bar{c},
\end{aligned}
$$

and that expressions (21) and (22) yield a rigid body displacement. If we wish to make $\varphi_{0}(z)$ and $\psi_{0}(z)$ vanish, we must impose three independent conditions, for example 


$$
\begin{aligned}
\varphi^{\prime}(a)-\overline{\varphi^{\prime}(a)} & =0, \\
\varphi(b) & =0,
\end{aligned}
$$

where the points $z=a$ and $z=b$ lie in $S$.

If however $\varphi_{0}(z)$ and $\psi_{0}(z)$ are assumed to be expressible in the forms given by equations (16) and (17), then by imposing only a single condition to make $\epsilon$ vanish, we can show as a consequence that $c$ also vanishes. In fact, in Section 3 , this particular feature of the method represents the key to the existence proof. Condition (20) is used to reduce $\epsilon$ to zero and the analysis then establishes that $c$ must also be zero.

Looking at this from another point of view, we can say that for $\varphi(z)$ and $\psi(z)$ to be expressible in the forms (16) and (17) and at the same time for $\varphi(z)$ and $\psi(z)$ to yield a rigid body translation, i.e.

$$
\varphi(z)=c, \quad \psi(z)=-\bar{c},
$$

$c$ must be zero. This means consequently that a non-zero rigid body translation alone is excluded by equations (16) and (17), and we note that this result is independent of condition (20).

The question now arises as to what particular functional form for $b_{m+1}[\omega(t)]$, subject to conditions (38) and (39), should be used. There are, in our opinion, two possible criteria that can be employed in such a determination. On the one hand, a mathematically very simple functional form may be desired, for example

$$
b_{m+1}[\omega(t)]=\frac{1}{2 \pi i} \oint_{\boldsymbol{\Gamma}}\left\{\frac{\omega(t) d t}{t^{2}}+\frac{\overline{\omega(t)}}{\bar{t}^{2}}\right\} .
$$

On the other hand, a functional form that is physically quite significant may be sought, for example,

$$
b_{m+1}[\omega(t)]=\varphi^{\prime}(0)-\overline{\varphi^{\prime}(0)} .
$$

Recalling equations (4) and (8), we see that expression (66) and condition (20) imply a zero rotation of an infinitesimal element of the body at the point $z=0$ for the case when the surface tractions on each of the inner contours are self-equilibrating.

In conclusion, we wish to reemphasize the vital importance of the existence proof in Section 3. We show that equations (12) and (13), subject to condition (11), must have a unique solution. This is extremely important from a practical point of view. In most cases, a closed form solution for $\omega(t)$ in equations (12) and (13) is impossible. A numerical approach therefore represents the alternative. In this case, the fact that a solution exists and is unique greatly simplifies the numerical procedure and assures at the same time that, from an analytical point of view, no peculiarities can arise. We might even speculate that the prospect of a numerical procedure was a prime motivating factor for Sherman in his derivation of a uniqueness and existence proof. 


\section{REFERENCES}

[1] Muskhelishvili, N. I., Some Basic Problems of the Mathematical Theory of Elasticity, P. Noordhoff Ltd., Groningen, Holland, 1953.

[2] Sherman, D. I., Sur la solution du second problème fondamental de la théorie statique plane de l'élasticité, Comptes Rendus Acad. Sci. URSS, 28 (1940) 25-31.

Pratt \& Whitney Aircraft

East Hartford, Conn.

Date Communicated: February 1, 1966 